Lung Alerts

\title{
Call for Lung Alerts
}

\section{J A Wedzicha, A Bhowmik, T Seemungal}

\section{An opportunity to contribute to the new Lung Alert feature in} Thorax.

n the January issue of Thorax we started a new feature called Lung

Alerts with the aim of increasing the educational value of Thorax. At a time of ever increasing numbers of medical publications and a vast range of journals publishing papers of interest to practitioners in respiratory medicine, we do not have the time to scan all the general and specialist journals for papers on respiratory topics. A number of very important papers are published in general medical or scientific journals, or in specialist journals such as those on oncology, paediatrics and epidemiology. Physicians and scientists may have varying access to these publications and thus may miss important new developments in basic science or clinical medicine.

We have therefore started to publish short reviews and alerts of papers that we have selected from these journals, printing the title of the paper with a short commentary of about 200 words which summarises the main message or learning point from the paper. These alerts will be attributed to the reviewer and may include a personal view on the paper, especially if the topic is controversial. The articles, usually original ones, are currently selected by our two Web Editors from papers published during the previous month and are then sent by email to the reviewers. We are keen to expand the bank of reviewers and are asking for volunteers from anywhere in the world to take part in the Lung Alert feature. We will usually suggest a paper relevant to your particular interest area, but we will also be interested to hear about any publications that you feel would be suitable for review. Please be sure to let us know the title of the paper before completing the review to avoid duplication of effort. We are especially keen to encourage younger physicians and scientists and doctors in training to come forward, although the Lung Alert series is not aimed exclusively at this group.

As the Alerts must be based on recent papers, we will need reviewers to respond with the completed review within 5 days from receipt of the commission. A prompt response from the reviewer is essential as we want this series to be as up to date and topical as possible. The Lung Alert will be published generally about two months after submission, and at present we are inserting them as "fillers" using space available at the end of articles. Links are provided to the main articles on our web site at www.thoraxjnl.com.

If you are interested in taking part then please contact one of our Web Editors at the email addresses given below, stating your interest areas. We would like to thank the reviewers who have already helped us to start the new feature and for their adherence to our deadlines. We know that they have all enjoyed writing them and are sure that you will find Lung Alerts of educational value, interesting and, of course-fun.

Thorax 2003;58:193

Authors' affiliations

J A Wedzicha, Editor in Chief

A Bhowmik, Web editor

(angshub@cheerful.com)

T Seemungal, Web editor

(TSeemungal@aol.com)

Correspondence to: Thorax Editorial Office, 17 Doughty Street, London WCIN 2PL, UK thorax@bmigroup.com 